\title{
Some properties of volume-biased weighted Weibull distribution
}

\author{
Zulfiqar Ahmed ${ }^{1}$ * ${ }^{\text {, Zahida Perveen }}{ }^{2}$, Munir Ahmad ${ }^{3}$ \\ ${ }^{1}$ Department of Computer Science, GIFT University, Gujranwala, Pakistan \\ 2Department of Mathematics, Lahore Garrison University, DHA Campus, Lahore, Pakistan \\ ${ }^{3}$ National College of Business Administration and Economics, Lahore, Pakistan
}

\section{A R T I C L E I N F O}

\section{Article history:}

Received 24 April 2017

Received in revised form

26 June 2017

Accepted 22 July 2017

\section{Keywords:}

Weighted distribution

Weibull distribution

Moments

Estimation

Recurrence relation

Entropy

Characterization

\begin{abstract}
A B S T R A C T
This paper introduces a new distribution based on the Weibull distribution, known as Volume biased Weighted Weibull Distribution (VWWD). We have discussed some statistical properties of this distribution in detail and obtained the mean, variance, moments, mode, coefficient of skewness, coefficient of kurtosis, reliability function, hazard function and the reverse hazard function. We have also provided results of entropies and characterization of VWWD. The parameters of this distribution are estimated by the maximum likelihood estimation method. At the end, we have compared this distribution with other well-known distributions and it is observed that VWWD fits better than those distributions.
\end{abstract}

(C) 2017 The Authors. Published by IASE. This is an open access article under the CC BY-NC-ND license (http://creativecommons.org/licenses/by-nc-nd/4.0/).

\section{Introduction}

Replication and randomization mostly occur on a large scale in traditional environmental theory. In many situations, observations are found in the nonreplicated, non-experimental and nonrandom classes. At this stage, the problems of model specification and data interpretation then acquire special importance and great concern. To solve these problems, the theory of weighted distributions offers a unifying method for these problems. For more detail of weighted distribution, see Perveen et al. (2016).

Let $f(x ; \theta)$ be the pdf of the random variable $x$ and $\theta$ be the unknown parameter. The weighted distribution is defined as;

$$
g(x ; \theta)=\frac{W(x) f(x ; \theta)}{E[W(x)]}
$$

where $x \in R, \theta>0$; where $w(x)$ is a weight function, defined as $w(x)=x^{m}$, where $m=3$, it is called as volume biased of order 3 .

Weibull Distribution is an important and wellknown distribution, which attracted statisticians, working in various fields of applied statistics as well as theory and methods in modern statistic due to its number of special features and ability to fit to data

\footnotetext{
* Corresponding Author.

Email Address: dr.zulfiqar.ahmed@hotmail.com (Z. Ahmed) https://doi.org/10.21833/ijaas.2017.08.025

2313-626X/C 2017 The Authors. Published by IASE.

This is an open access article under the CC BY-NC-ND license

(http://creativecommons.org/licenses/by-nc-nd/4.0/)
}

related to various fields like as life testing, biology, ecology, economics, hydrology, engineering and business administration. This distribution is one of the members of the family of extreme value distributions. For more detail see Perveen et al. (2016).

Provost et al. (2009) introduced some properties of three parameter weighted Weibull distribution. He defined the probability density function as

$\mathrm{f}(\mathrm{x} ; \mathrm{k}, \xi, \theta)=\frac{\mathrm{k} \theta \frac{\xi}{\mathrm{k}+1} \mathrm{x}^{\xi+\mathrm{k}-1} \mathrm{e}^{-\theta \mathrm{x}^{\mathrm{k}}}}{\Gamma\left(1+\frac{\xi}{\mathrm{k}}\right)}, \quad \mathrm{x}>0$

with $\xi+\mathrm{k}>0$, and with shape parameter $\xi$.

\subsection{Volume-biased weighted Weibull distribution}

Theorem. 1: Let $X$ be a non - negative random variable, then the following relationship between Eq. 1 and the weight function $\mathrm{w}(\mathrm{x})$ can be defined as $g(x ; \xi, \theta, k)=\frac{w(x) f(x)}{\int_{0}^{\infty} w(x) f(x) d x}$ where $w(x)$ is the first weight, $w(x)=x^{3}$ and $f(x)$ is defined in Eq. 2.

Proof: Suppose $\mathrm{X}$ has a density function $\mathrm{g}(\mathrm{x} ; \xi, \mathrm{k}, \theta)$ with unknown parameters $\xi, \theta, \mathrm{k}$. Using Eq. 1 and Eq. 2, the corresponding distribution, named as volume biased weighted Weibull distribution is of the type:

$\mathrm{g}(\mathrm{x} ; \xi, \theta, \mathrm{k})=\frac{\mathrm{k} \theta^{1+\frac{3}{\mathrm{k}}+\frac{\xi}{\mathrm{k}} \mathrm{x}^{\xi+\mathrm{k}+2} \mathrm{e}^{-\theta \mathrm{x}^{\mathrm{k}}}}}{\Gamma\left(1+\frac{3+\xi}{\mathrm{k}}\right)} \quad \xi, \theta, \mathrm{k}>0,0<\mathrm{x}<$

$\infty$ 
where $\xi$ and $k$ are shape parameters and $\theta$ is the scale parameter.

Fig. 1 shows the graphs of VWWD density function for various values of parameters.

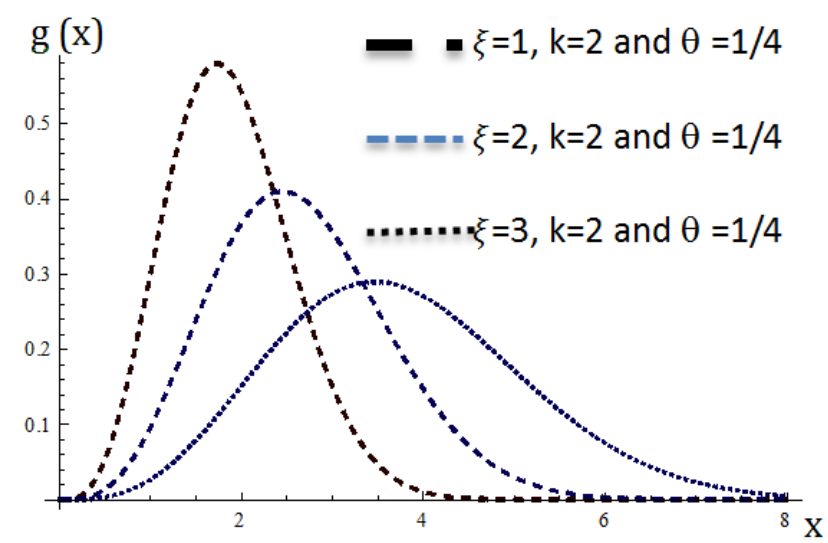

Fig. 1: Probability density function of VWWD for the indicated values of $\xi$, $k$ and $\theta$

Theorem. 2: Let $\mathrm{X}$ be a non-negative random variable with the probability density function 3 , then the cumulative distribution function (cdf) is defined as:

$\mathrm{G}(\mathrm{x}, \xi, \theta, \mathrm{k})=1-\frac{1}{\Gamma\left(1+\frac{3+\xi}{\mathrm{k}}\right)} \Gamma\left(\theta \mathrm{x}^{\mathrm{k}}, 1+\frac{3+\xi}{\mathrm{k}},\right), \xi, \mathrm{k}, \theta>0$

where $\Gamma(\mathrm{a}, \quad \mathrm{x})=\int_{\mathrm{x}}^{\infty} \mathrm{y}^{\mathrm{a}-1} \mathrm{e}^{-\mathrm{y}} \mathrm{dy} \quad$ represents an incomplete gamma function.

Proof: Trivially using integration by parts we have the requested form Eq. 4.

Fig. 2 shows the graph of Distribution Function.

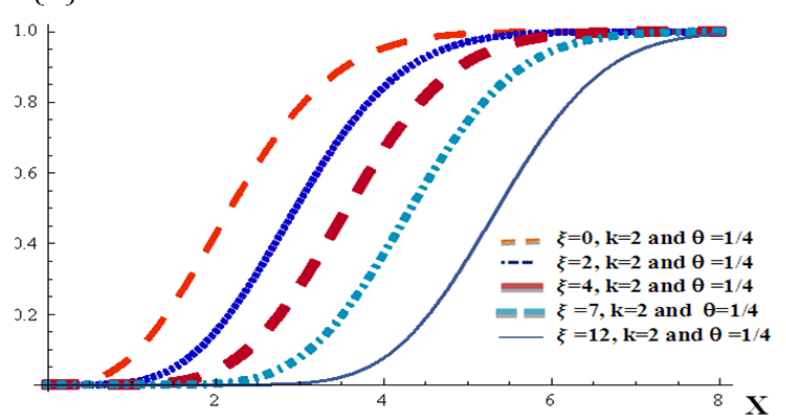

Fig. 2: Distribution function of VWWD for the indicated values of $\theta, \xi$ and $\mathrm{k}$

\subsection{The survival function of VWWD}

The survival function is an important measure in reliability studies. The survival function for VWWD is

$\mathrm{S}(\mathrm{x})=1-\mathrm{G}(\mathrm{x})=\frac{\Gamma\left(\theta \mathrm{x}^{\mathrm{k}}, 1+\frac{3+\xi}{\mathrm{k}}\right)}{\Gamma\left(1+\frac{3+\xi}{\mathrm{k}}\right)}, \xi, \mathrm{k}, \theta>0$

Fig. 3 shows the Graph of Survival Function.

\subsection{The hazard rate function of VWWD}

The hazard function is the instant level of failure at a certain time. Characteristics of a hazard function are normally related with definite products and applications. Different hazard functions are displayed with different distribution models. Nadarajah and Kotz (2004) pointed out some properties of Hazard rate. Dara (2011) evaluated the reliability measures of weighted distributions.

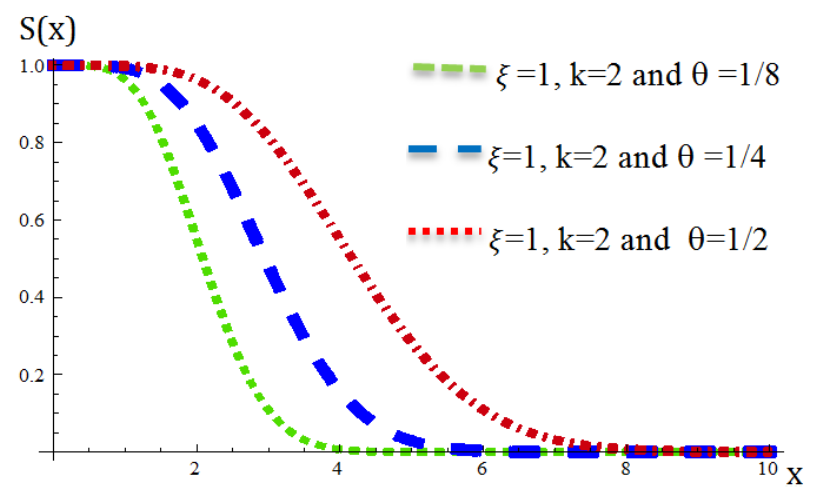

Fig. 3: Survival function of VWWD for the indicated values of $\theta, \xi$ and $\mathrm{k}$

Theorem. 3: Suppose X be a non-negative random variable with the probability density function then hazard rate is defined by $h(x)=\frac{g(x)}{S(x)}$, where $S(x)$ is the survival function.

Proof: By putting values of pdf and $S(x)$ in above formula, at $\mathrm{c}=1$ and $\lambda=1$, hazard rate will be

$h(x)=\frac{k \theta^{1+\frac{3}{k}+\frac{\xi}{k}} x^{\xi+k+2} e^{-\theta x^{k}}}{\Gamma\left(1+\frac{3+\xi}{k}, \theta x^{k}\right)}, \xi, k, \theta>0$.

Fig. 4 shows hazard rate function graphically.

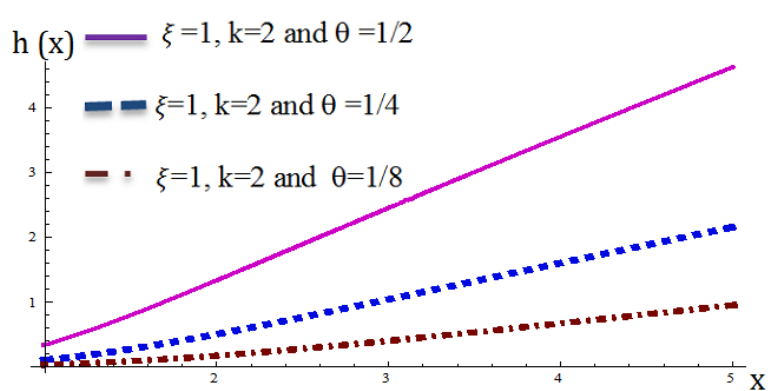

Fig. 4: Hazard rate function of VWWD for the indicated values of $\xi$, $\mathrm{k}$ and $\theta$

Corollary 1: Let $X$ be a non - negative random variable then Reverse hazard function is the quotient of pdf and hazard rate e.g., $r(x)=\frac{g(x)}{G(x)}$.

Proof: By putting required values in above formula, we have the following,

$r(x)=\frac{\mathrm{g}(\mathrm{x})}{\mathrm{G}(\mathrm{x})}=\frac{\mathrm{k} \theta^{1+\frac{3}{\mathrm{k}}+\frac{\xi}{\mathrm{k}} \mathrm{x}^{\xi+\mathrm{k}+2} \mathrm{e}^{-\theta} \mathrm{x}^{\mathrm{k}}}}{\Gamma\left(1+\frac{3+\xi}{\mathrm{k}}\right)-\Gamma\left(1+\frac{3+\xi}{\mathrm{k}}, \theta \mathrm{x}^{\mathrm{k}}\right)} \theta, \mathrm{k}, \xi>0$

Fig. 5 shows Graph of Reverse Hazard Function.

\section{Moments ( ${ }^{\text {th }}$ moments about zero)}

Suppose $\mathrm{X}$ is a random variable with pdf $\mathrm{g}(\mathrm{x})$ as given in Eq. 3. Then using gamma function, $r$ th moment is easily expressed as 


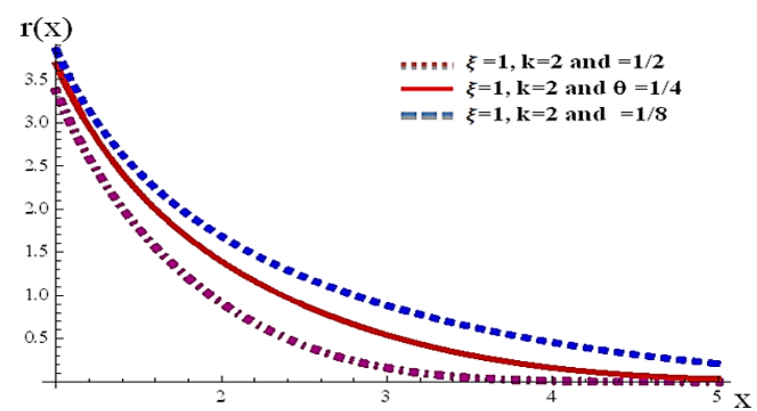

Fig. 5: Reverse hazard rate function of VWWD for the indicated values of $\xi, \mathrm{k}$ and $\theta$

$\mu_{r}^{\prime}=E\left(x^{r}\right)=\frac{\theta^{\frac{-r}{k}}}{r\left(1+\frac{3+\xi}{k}\right)} r\left(1+\frac{3+\xi+r}{k}\right)$

The four moments can be obtained by putting $r=$ $1,2,3,4$ about the mean are:

$\mu_{1}=0$

$\mu_{2}=\frac{\theta^{\frac{-3}{k}} \mathrm{r}\left(1+\frac{5+\xi}{k}\right)}{r\left(1+\frac{3+\xi}{k}\right)}-\frac{\theta^{\frac{-2}{k}} r\left(1+\frac{5+\xi}{k}\right)^{2}}{r\left(1+\frac{3+\xi}{k}\right)^{2}}$

$\mu_{3}=\frac{\theta^{\frac{-3}{\mathrm{k}}} \mathrm{r}\left(1+\frac{6+\xi}{\mathrm{k}}\right)}{\mathrm{r}\left(1+\frac{3+\xi}{\mathrm{k}}\right)}-\frac{3 \theta^{\frac{-3}{\mathrm{k}}} \mathrm{r}\left(1+\frac{4+\xi}{\mathrm{k}}\right) \mathrm{r}\left(1+\frac{5+\xi}{\mathrm{k}}\right)}{\left[\mathrm{r}\left(1+\frac{3+\xi}{\mathrm{k}}\right)\right]^{2}}+\frac{2 \theta^{\frac{-3}{\mathrm{k}}} \mathrm{r}\left(1+\frac{5+\xi}{\mathrm{k}}\right)^{3}}{\mathrm{r}\left(1+\frac{3+\xi}{\mathrm{k}}\right)^{3}}$

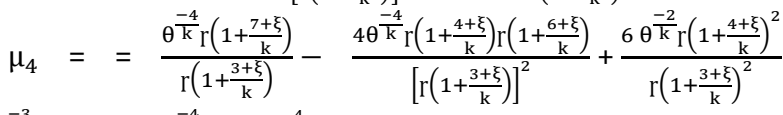

$\frac{\theta^{\frac{-3}{\mathrm{k}}} \mathrm{r}\left(1+\frac{5+\xi}{\mathrm{k}}\right)}{\mathrm{r}\left(1+\frac{3+\xi}{\mathrm{k}}\right)}-\frac{\theta^{\frac{-4}{\mathrm{k}}} \mathrm{r}\left(1+\frac{5+\xi}{\mathrm{k}}\right)^{4}}{\mathrm{r}\left(1+\frac{3+\xi}{\mathrm{k}}\right)^{4}}$

Table 1 shows the Measure of Coefficient of Skewness and Kurtosis for VWWD. From Table 1 it is clear that VWWD is almost symmetrical and platykurtic for $2.9 \leq \mathrm{k} \leq 3.3$.

Table 1: Measure of Coefficient of Skewness and Kurtosis

\begin{tabular}{cccccc}
\multicolumn{7}{c}{ for VWWD } \\
\hline $\mathrm{k}$ & 2.9 & 3.0 & 3.1 & 3.2 & 3.3 \\
\hline$\theta$ & 0.5 & 0.5 & 0.5 & 0.5 & 0.5 \\
$\xi$ & 1.0 & 1.0 & 1.0 & 1.0 & 1.0 \\
$\sqrt{\beta_{1}}$ & 0.068 & 0.042 & 0.026 & 0.007 & 0.001 \\
$\beta_{2}$ & 2.889 & 2.986 & 2.991 & 2.999 & 3,001 \\
\hline
\end{tabular}

\section{Limit and mode of the function}

The limit of density function given in Eq. 3 is as follows:

$\operatorname{Lim}_{x \rightarrow 0} g(x ; k, \theta, \xi)=x \rightarrow 0 \frac{k \theta^{1+\frac{3}{k}+\frac{\xi}{k}} x^{\xi+k+2} e^{-\theta x^{k}}}{\Gamma\left(1+\frac{3+\xi}{k}\right)}=0$

$\operatorname{Lim}_{x \rightarrow 0} \mathrm{~g}(\mathrm{x} ; \mathrm{k}, \theta, \xi)=\frac{\mathrm{k} \theta^{1+\frac{3}{\mathrm{k}}+\frac{\xi}{\mathrm{k}}}}{\Gamma\left(1+\frac{3+\xi}{\mathrm{k}}\right)} \mathrm{x} \rightarrow \infty \mathrm{x}^{2+\xi+\mathrm{k}} \mathrm{e}^{-\theta \mathrm{x}^{\mathrm{k}}}=0$.

\section{Mode of VWWD}

Mode of Eq. 3 can be found by solving equation $\frac{\partial}{\partial \mathrm{x}} \log \left(\mathrm{g}(\mathrm{x} ; \mathrm{k}, \theta, \xi)=0\right.$, we have mode $=\left\langle\frac{\xi+\mathrm{k}+2}{\theta \mathrm{k}}\right\rangle \frac{1}{\mathrm{k}}$.

Tables 2, 3, and 4 shows the mode of VWWD for given values of $\theta, \xi$ and $\mathrm{k}$.

\section{Moment generating function (MGF)}

The moment generating function of VWWD is given as $\mathrm{M}_{\mathrm{X}}(\mathrm{t})=\int_{0}^{\infty} \mathrm{e}^{\mathrm{tx}} \mathrm{g}(\mathrm{x}) \mathrm{dx}$.
Using value of $\mathrm{g}(\mathrm{x})$ from Eq. 3 and after some simplification we get

$\mathrm{M}_{\mathrm{X}}(\mathrm{t})=\sum_{\mathrm{i}=1}^{\infty} \frac{\mathrm{t}^{\mathrm{i}}}{\mathrm{i} !} \frac{\theta^{\frac{-\mathrm{i}}{\mathrm{k}}}}{\Gamma\left(1+\frac{3+\xi}{\mathrm{k}}\right)} \Gamma\left(1+\frac{3+\mathrm{i}+\xi}{\mathrm{k}}\right), \mathrm{K}>0$

\section{Information generating function}

The moment generating function of VWWD can be written as

$\mathrm{T}(\mathrm{s})=\frac{\mathrm{k}^{\mathrm{s}-1} \theta^{\frac{\mathrm{s}-1}{\mathrm{k}}}}{\Gamma\left(1+\frac{3+\xi}{\mathrm{k}}\right)^{\mathrm{s}} \mathrm{s}^{\mathrm{s}+\frac{\mathrm{s} \xi+2 s+1}{k}}} \Gamma\left(\mathrm{s}+\frac{\mathrm{s} \xi+2 \mathrm{~s}+1}{\mathrm{k}}\right)$.

Table 2: Mode of VWWD for values $\xi=1, \mathrm{k}=2$

\begin{tabular}{ccccccc}
\hline$\theta$ & 0.500 & 0.450 & 0.400 & 0.350 & 0.300 & 0.250 \\
\hline Mode & 1.662 & 1.342 & 1.832 & 2.001 & 2.149 & 2.532 \\
\hline
\end{tabular}

Table 3: Mode of VWWD for values $\theta=0.5, \xi=1$

\begin{tabular}{ccccccc}
\hline $\mathrm{k}$ & 2.900 & 3.000 & 3.100 & 3.200 & 3.300 & 3.400 \\
\hline Mode & 1.406 & 1.386 & 1.357 & 1.352 & 1.336 & 1.322 \\
\hline
\end{tabular}

Table 4: Mode of VWWD for values $\theta=0.5, \mathrm{k}=3$

\begin{tabular}{ccccccc}
\hline$\xi$ & 0.500 & 0.450 & 0.400 & 0.350 & 0.300 & 0.250 \\
\hline Mode & 1.326 & 1.386 & 1.442 & 1.493 & 1.542 & 1.587 \\
\hline
\end{tabular}

\section{Estimation of parameters}

Maximum likelihood (ML) Estimation is used to estimate the parameters of VWWD. If $\mathrm{X}_{1}, \mathrm{X}_{2} \ldots \ldots \mathrm{X}_{\mathrm{n}}$ be a random sample from a population having pdf $\mathrm{g}(\mathrm{x} \mid \mathrm{k}, \theta, \xi)$ the likelihood function of VWWD distribution can be defined as $\mathrm{L}$ $\left(\theta, \xi, k ; x_{1}, x_{2}, \ldots, x_{n}\right)=\prod_{i=1}^{n} g\left(x_{i}\right)$.

Let $\mathrm{x}_{1}, \mathrm{x}_{2}, \ldots, \mathrm{x}_{\mathrm{n}}$ are the independent observations, then the log likelihood function of the distribution is

$\mathrm{L}\left(\theta, \xi, \mathrm{k} ; \mathrm{x}_{1}, \mathrm{x}_{2}, \ldots, \mathrm{x}_{\mathrm{n}}\right)=\sum_{\mathrm{i}=1}^{\mathrm{n}} \log \left(\mathrm{g}\left(\mathrm{x}_{\mathrm{i}} ; \theta, \xi, \mathrm{k}\right)=\mathrm{n} \log \mathrm{k}+\mathrm{n}\right.$ $\left(1+\frac{3}{\mathrm{k}}+\frac{\xi}{\mathrm{k}}\right) \log \theta$

$-\mathrm{nlog} \Gamma\left(1+\frac{3+\xi}{\mathrm{k}}\right)+(\mathrm{k}+\xi+2) \sum_{\mathrm{i}=1}^{\mathrm{n}} \log \mathrm{x}_{\mathrm{i}}-\theta \sum_{\mathrm{i}=1}^{\mathrm{n}} \mathrm{x}_{\mathrm{i}}^{\mathrm{k}}(13)$

ML estimates can be found by solving Equations

$\frac{\partial \mathrm{l}(\theta, \bar{\xi}, \mathrm{k} ; \underline{\mathbf{x}})}{\partial \xi}=0, \frac{\partial \mathrm{l}(\theta, \bar{\xi}, \mathbf{k} ; \underline{\mathbf{x}})}{\partial \mathrm{k}}=0, \frac{\partial \mathrm{l}(\theta, \xi, \mathrm{k} ; \underline{\mathbf{x}})}{\partial \theta}=0$

$\frac{\mathrm{n}}{\widehat{\mathrm{k}}} \log (\hat{\theta})+\sum_{\mathrm{i}=1}^{\mathrm{n}} \log \left(\mathrm{x}_{\mathrm{i}}\right)-\frac{\mathrm{n}}{\widehat{\mathrm{k}}} \Psi^{(0)}\left(1+\frac{3+\widehat{\xi}}{\widehat{\mathrm{k}}}\right)=0$,

Where

$\Psi^{(0)}(\mathrm{z})=\frac{\Gamma^{\prime}(\mathrm{z})}{\Gamma(\mathrm{z})}$

$\frac{\mathrm{n}\left(\widehat{\mathrm{k}}-(3+\hat{\xi}) \log (\widehat{\theta})+(3+\hat{\xi}) \Psi^{(0)}\left(1+\frac{3+\hat{\xi}}{\hat{\mathrm{k}}}\right)\right)}{\widehat{\mathrm{k}}^{2}}+\sum_{\mathrm{i}=1}^{\mathrm{n}} \log \left(\mathrm{x}_{\mathrm{i}}\right)-$

$\widehat{\theta} \sum_{\mathrm{i}=1}^{\mathrm{n}} \mathrm{x}_{\mathrm{i}}^{\widehat{\mathrm{k}}} \log \left(\mathrm{x}_{\mathrm{i}}\right)=0$

$n\left(1+\frac{3}{\hat{k}}+\frac{\xi}{\hat{k}}\right) \cdot \frac{1}{\hat{\theta}}-\sum_{i=1}^{n} x_{i}^{\widehat{k}}=0$

Eqs. 14, 15, and 16 are nonlinear equations and can be solved through Mathematica software.

Asymptotic variance-covariance matrix is the inverse of $\mathrm{I}(\theta, \mathrm{k}, \xi)=-\mathrm{E}(\mathrm{H}(\mathrm{X}))$ 
$\mathrm{H}(\mathrm{X})=\left(\begin{array}{lll}\frac{\partial^{2}(\log (\mathrm{g}(\mathrm{X} ; \theta, \mathrm{k}, \xi)}{\partial \xi^{2}} & \frac{\partial^{2}(\log (\mathrm{g}(\mathrm{X} ; \theta, \mathrm{k}, \xi))}{(\partial \xi \partial \mathrm{k})} & \frac{\partial^{2}(\log (\mathrm{g}(\mathrm{X} ; \theta, \mathrm{k}, \xi))}{(\partial \xi \partial \theta)} \\ \frac{\partial^{2}(\log (\mathrm{g}(\mathrm{X} ; \theta, \mathrm{k}, \xi))}{(\partial \mathrm{k} \partial \xi)} & \frac{\partial^{2}(\log (\mathrm{g}(\mathrm{X} ; \theta, \mathrm{k}, \xi))}{\partial \mathrm{k}^{2}} & \frac{\partial^{2}(\log (\mathrm{g}(\mathrm{X} ; \theta, \mathrm{k}, \xi))}{(\partial \mathrm{k} \partial \theta)} \\ \frac{\partial^{2}(\log (\mathrm{g}(\mathrm{X} ; \theta, \mathrm{k}, \xi))}{(\partial \theta \partial \xi)} & \frac{\partial^{2}(\log (\mathrm{g}(\mathrm{X} ; \theta, \mathrm{k}, \xi)}{(\partial \theta \partial \mathrm{k})} & \frac{\partial^{2}(\log (\mathrm{g}(\mathrm{X} ; \theta, \mathrm{k}, \xi))}{\partial \theta^{2}}\end{array}\right)$

where,

$$
\begin{aligned}
& \frac{\partial^{2}(\log (\mathrm{g}(\mathrm{X} ; \theta, \mathrm{k}, \xi))}{\partial \xi^{2}}=\frac{-\mathrm{n} \Psi^{(1)}\left(1+\frac{3+\xi}{\mathrm{k}}\right)}{\mathrm{k}^{2}}, \\
& \frac{\partial^{2}(\log (\mathrm{g}(\mathrm{X} ; \theta, \mathrm{k}, \xi))}{\partial \mathrm{k}^{2}}=\frac{-\mathrm{n}}{\mathrm{k}^{2}}-\frac{2 \mathrm{n}(3+\xi)}{\mathrm{k}^{3}} \log (\theta)-\theta \sum_{\mathrm{i}=1}^{\mathrm{n}} \mathrm{x}_{\mathrm{i}}^{\mathrm{k}} \log \left(\mathrm{x}_{\mathrm{i}}\right)^{2} \\
& -\frac{2 \mathrm{n}}{\mathrm{k}^{3}}(3+\xi) \Psi^{(0)}\left(1+\frac{3+\xi}{\mathrm{k}}\right)-\frac{\mathrm{n}(3+\xi)^{2}}{\mathrm{k}^{4}} \Psi^{(1)}\left(1+\frac{3+\xi}{\mathrm{k}}\right), \\
& \frac{\partial^{2}(\log (\mathrm{g}(\mathrm{X} ; \theta, \mathrm{k}, \xi))}{\partial \theta^{2}}=\frac{-\mathrm{n}}{\theta^{2}}\left(1+\frac{3}{\mathrm{k}}+\frac{\xi}{\mathrm{k}}\right) \text {, } \\
& \frac{\partial^{2}(\log (\mathrm{g}(\mathrm{X} ; \theta, \mathrm{k}, \xi))}{(\partial \theta \partial \mathrm{k})}=\frac{\partial^{2}(\log (\mathrm{g}(\mathrm{X} ; \theta, \mathrm{k}, \xi))}{(\partial \mathrm{k} \partial \theta)}=\frac{\mathrm{n}}{\theta}\left(\frac{-3}{\mathrm{k}^{2}}-\frac{\xi}{\mathrm{k}^{2}}\right)- \\
& \sum_{\mathrm{i}=1}^{\mathrm{n}} \mathrm{x}_{\mathrm{i}}^{\mathrm{k}} \log \left(\mathrm{x}_{\mathrm{i}}\right) \text {, } \\
& \frac{\partial^{2}(\log (\mathrm{g}(\mathrm{X} ; \theta, \mathrm{k}, \xi))}{(\partial \xi \partial \theta)}=\frac{\partial^{2}(\log (\mathrm{g}(\mathrm{X} ; \theta, \mathrm{k}, \xi))}{(\partial \theta \partial \xi)}=\frac{\mathrm{n}}{\theta \mathrm{k}^{\prime}} \\
& \frac{\partial^{2}(\log (\mathrm{g}(\mathrm{X} ; \theta, \mathrm{k}, \xi))}{(\partial \xi \partial \mathrm{k})}=\frac{\partial^{2}(\log (\mathrm{g}(\mathrm{X} ; \theta, \mathrm{k}, \xi))}{(\partial \mathrm{k} \partial \xi)},=\frac{-\mathrm{n}}{\mathrm{k}^{2}} \log (\theta)+\frac{\mathrm{n}}{\mathrm{k}^{2}} \Psi^{(0)}(1+ \\
& \left.\frac{3+\xi}{\mathrm{k}}\right)-\frac{\mathrm{n}}{\mathrm{k}^{3}}(3+\xi) \Psi^{(1)}\left(1+\frac{3+\xi}{\mathrm{k}}\right)
\end{aligned}
$$

\section{Recurrence relation of volume biased weighted Weibull distribution}

Theorem. 4: Let $X$ be the random variable on its support $(0, \infty)$. Then recurrence relation through conditional moments for all $t>0$

$$
\mathrm{E}\left(\mathrm{X}^{\mathrm{nk}} \mid \mathrm{X}>\mathrm{t}\right)=\frac{\theta^{\mathrm{n}+\frac{3+\xi}{\mathrm{k}} \mathrm{t}^{\mathrm{nk}+(1+\xi)} \mathrm{e}^{-\theta \mathrm{t}^{\mathrm{k}}}}}{\Gamma\left(1+\frac{3+\xi}{\mathrm{k}}, \theta \mathrm{t}^{\mathrm{k}}\right) \theta^{\mathrm{n}}}+\frac{\left(\mathrm{n}+\frac{3+\xi}{\mathrm{k}}\right)}{\theta} \mathrm{E}\left(\mathrm{X}^{(\mathrm{n}-1) \mathrm{k}} \mid \mathrm{X}>\right.
$$$$
\text { t) }
$$

where $\xi, \theta, \mathrm{k}>0$ and $\mathrm{n} \in \mathrm{z}^{+}$.

Proof: Let X be the volume biased weighted Weibull distribution. Then

$$
\begin{aligned}
& E\left(X^{n k} \mid X>t\right)=\frac{1}{G_{1}(t)} \int_{t}^{\infty} x^{n k} g(x) d x \\
& \text { Using } \bar{G}(t)=\frac{\Gamma\left(1+\frac{3+\xi}{k}, \theta t^{k}\right)}{\Gamma\left(1+\frac{3+\xi}{k}\right)} \\
& =\frac{k \theta^{1+\frac{3}{k}+\frac{\xi}{k}}}{\Gamma\left(1+\frac{3+\xi}{k}, \theta t^{k}\right)} \int_{t}^{\infty} x^{n k+\xi+k} e^{-\theta x^{k}} d x
\end{aligned}
$$

using the transformation $\theta \mathrm{x}^{\mathrm{k}}=\mathrm{u}, \mathrm{k} \theta \mathrm{x}^{\mathrm{k}-1} \mathrm{dx}=$ $\mathrm{du}$ and $\theta \mathrm{t}^{\mathrm{k}}<\mathrm{u}<\infty$

$$
\begin{aligned}
& =\int_{\theta t^{\mathrm{k}}}^{\infty}\left(\frac{\mathrm{u}}{\theta}\right)^{\mathrm{n}}\left(\frac{\mathrm{u}}{\theta}\right)^{\frac{\xi+\mathrm{k}}{\mathrm{k}}} \mathrm{e}^{-\mathrm{u}} \cdot \frac{\mathrm{du}}{\theta\left(\frac{\mathrm{u}}{\theta}\right)^{\frac{\mathrm{k}-1}{\mathrm{k}}}}= \\
& \frac{\theta^{\frac{3}{\mathrm{k}^{+}}+\frac{\xi}{\mathrm{k}}}}{\Gamma\left(1+\frac{3+\xi}{\mathrm{k}}, \theta \mathrm{t}^{\mathrm{k}}\right) \theta^{\mathrm{n}}} \int_{\theta t^{\mathrm{k}}}^{\infty}(\mathrm{u})^{\mathrm{n}+\frac{3}{\mathrm{k}}+\frac{\xi}{\mathrm{k}}} \mathrm{e}^{-\mathrm{u}} \mathrm{du}
\end{aligned}
$$

using integration by parts, we get:

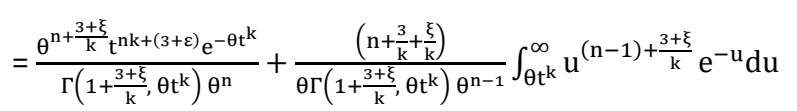

After some simplification, we will obtain Eq. 24.

\section{Entropy}

Entropy is considered as a major tool in every field of science and technology. In Statistics entropy is considered as an amount of incredibility. Shanon entropy is defined as $\mathrm{h}(\mathrm{X})$ of a continuous random variable $\mathrm{X}$ with a density function $\mathrm{f}(\mathrm{x})$ (Gradštejn and Ryžik, 2007)

$\mathrm{h}(\mathrm{X})=\mathrm{E}[-\log (\mathrm{f}(\mathrm{x}))]$

$\mathrm{h}[\mathrm{g}(\mathrm{x} ; \theta, \xi, \mathrm{k})]=\mathrm{E}[-\log \mathrm{g}(\mathrm{x} ; \theta, \xi, \mathrm{k})]$

$=\mathrm{E}\left[-\log \left\{\frac{\mathrm{k} \theta^{1+\frac{3}{\mathrm{k}}+\frac{\xi}{\mathrm{k}} \mathrm{x}^{\xi+\mathrm{k}+2}} \mathrm{e}^{-\theta^{\mathrm{x}^{\mathrm{k}}}}}{\Gamma\left(1+\frac{3+\xi}{\mathrm{k}}\right)}\right\}\right]$

$=\mathrm{E}\left[\theta \mathrm{x}^{\mathrm{k}}-\log (\mathrm{k})-(\mathrm{k}+\xi+2) \log (\mathrm{x})-\left(1+\frac{3}{\mathrm{k}}+\right.\right.$

$\left.\left.\frac{\xi}{\mathrm{k}}\right) \log (\theta)+\log \Gamma\left(1+\frac{3+\xi}{\mathrm{k}}\right)\right]$

$=\mathrm{E}\left(\theta \mathrm{x}^{\mathrm{k}}\right)-\log (\mathrm{k})-(\mathrm{k}+\xi+2) \mathrm{E} \log (\mathrm{x})-\left(1+\frac{3}{\mathrm{k}}+\right.$

$\left.\frac{\xi}{\mathrm{k}}\right) \log (\theta)+\log \Gamma\left(1+\frac{3+\xi}{\mathrm{k}}\right)$

$=\log \frac{\Gamma\left(1+\frac{3+\xi}{k}\right)}{k}-\left(1+\frac{3}{k}+\frac{\xi}{k}\right) \log (\theta)-(\xi+k+2) E \log (x)+$ $\mathrm{E}\left[\theta \mathrm{x}^{\mathrm{k}}\right]$.

$\operatorname{Elog}(\mathrm{x})=\frac{1}{\mathrm{k}} \frac{1}{\Gamma\left(1+\frac{3+\xi}{\mathrm{k}}\right)} \int_{0}^{\infty} \log \left(\frac{\mathrm{t}}{\theta}\right) \cdot \mathrm{t}^{\frac{1+\xi}{\mathrm{k}}} \mathrm{e}^{-\mathrm{t}} \mathrm{dt}=$

$\frac{1}{\mathrm{k}} \frac{1}{\Gamma\left(1+\frac{3+\xi}{\mathrm{k}}\right)}\left[\int_{0}^{\infty} \log \mathrm{t} \cdot \mathrm{t}^{\frac{3+\xi}{\mathrm{k}}} \mathrm{e}^{-\mathrm{t}} \mathrm{dt}-\int_{0}^{\infty} \log \theta \mathrm{t}^{\frac{3+\xi}{\mathrm{k}}} \mathrm{e}^{-\mathrm{t}} \mathrm{dt}\right]$

$\int_{0}^{\infty} \log \mathrm{x} \mathrm{x}^{\gamma-1} \mathrm{e}^{-\mathrm{x}} \mathrm{dx}=\Gamma^{\prime}(\gamma)$

$\operatorname{Elog}(\mathrm{x})=\frac{1}{\mathrm{k}} \frac{1}{\Gamma\left(1+\frac{3+\xi}{\mathrm{k}}\right)}\left[\Gamma^{\prime}\left(1+\frac{3+\xi}{\mathrm{k}}\right)-\log \theta \Gamma\left(1+\frac{3+\xi}{\mathrm{k}}\right)\right]$.

$\mathrm{E}\left[\theta \mathrm{x}^{\mathrm{k}}\right]=\frac{\mathrm{k} \theta^{2+\frac{3}{\mathrm{k}}+\frac{\xi}{\mathrm{k}}}}{\Gamma\left(1+\frac{3+\xi}{\mathrm{k}}\right)} \Gamma\left(2+\frac{3+\xi}{\mathrm{k}}\right)$.

Putting Eq. 28 and Eq. 29 in Eq. 27 to get

$\mathrm{h}[\mathrm{g}(\mathrm{x} ; \xi, \mathrm{k}, \theta)]=\log \frac{\Gamma\left(1+\frac{3+\xi}{\mathrm{k}}\right)}{\mathrm{k}}-\left(1+\frac{3}{\mathrm{k}}+\frac{\xi}{\mathrm{k}}\right) \log (\theta)$

$-(\xi+\mathrm{k}+2) \frac{1}{\mathrm{k}} \frac{1}{\Gamma\left(1+\frac{3+\xi}{\mathrm{k}}\right)}\left(\Gamma^{\prime}\left(1+\frac{3+\xi}{\mathrm{k}}\right)-\log \theta \Gamma\left(1+\frac{3+\xi}{\mathrm{k}}\right)\right)+$

$\frac{\mathrm{k} \theta^{2+\frac{3}{\mathrm{k}}+\frac{\xi}{\mathrm{k}}}}{\Gamma\left(1+\frac{3+\xi}{\mathrm{k}}\right)} \Gamma\left(2+\frac{3+\xi}{\mathrm{k}}\right)$

where $\xi, \mathrm{k}, \theta>0$.

Alfred Renyi entropy is usually known as the generalized procedure of Shannon entropy. The Renyi entropy is named after. It is useful in ecology and statistics. It is defined as

$\left.\operatorname{IR}(\beta)=\frac{1}{1-\beta} \log \left(\int_{0}^{\infty} \mathrm{g}^{\beta}(\mathrm{x}) \mathrm{dx}\right) \beta>0, \beta \neq 1\right)$

Putting value of $\mathrm{g}(\mathrm{x})$ from Eq. 3 in above equation, we get:

$\operatorname{IR}(\beta)=\frac{1}{1-\beta} \log \left[\int_{0}^{\infty}\left[\frac{\mathrm{k} \theta^{1+\frac{\xi}{\mathrm{k}}+\frac{3}{\mathrm{k}} \mathrm{\xi}+\mathrm{k}+2} \mathrm{e}^{-\theta^{\mathrm{x}^{\mathrm{k}}}}}{\Gamma\left(1+\frac{3+\xi}{\mathrm{k}}\right)}\right]^{\beta}\right]$

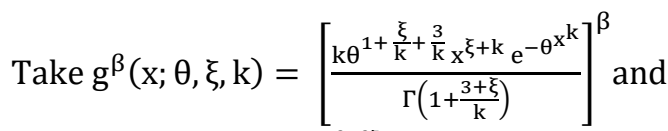
$\int_{0}^{\infty} g^{\beta}(x ; \theta, \xi, k) d x=\frac{k^{\beta} \theta^{\beta+\frac{\beta}{k}+\frac{\beta \xi}{k}}}{\left[\Gamma\left(1+\frac{3+\xi}{k}\right)\right]^{\beta}} \int_{0}^{\infty} x^{k \beta+\beta \xi+2 \beta} e^{-\beta \theta^{x^{k}}} d x$. $\operatorname{IR}(\beta)=\frac{\beta-1}{\mathrm{k}} \log \theta+\log \Gamma\left(\beta+\frac{3+\beta \xi}{\mathrm{k}}\right)-\log \mathrm{k}-\left(\beta+\frac{3}{\mathrm{k}}+\right.$ $\left.\frac{\beta \xi}{\mathrm{k}}\right) \log \beta-\beta \log \Gamma\left(1+\frac{3+\xi}{\mathrm{k}}\right)$. 


\section{Numerical example}

See for data set used by Silverman (1986) (i.e., the buffalo snowfall data set) given by Table 5 . In Table 5, the approximations of the parameters are specified. For goodness-of-fit statistics AndersonDarling and Cramer-von Mises tests have been used, the weighted Weibull model proposes the best fitting shown by the Fig. 6 and Fig. 7.

Table 5: Parameters' estimates and goodness-of-fit statistics

\begin{tabular}{|c|c|c|c|c|c|c|c|c|}
\hline Distributions & $\widehat{\theta}$ & $\hat{\mathrm{k}}$ & $\hat{\xi}$ & $\hat{\lambda}$ & $\widehat{\alpha}$ & $\widehat{\beta}$ & $\mathrm{A}_{0}^{2}$ & $\mathrm{~W}_{0}^{2}$ \\
\hline Two parameter Weibull & $3.37477 \times 10^{-8}$ & 3.8338 & - & 88.898 & - & - & 0.3063 & 0.0454 \\
\hline Size biased Rayleigh & - & - & - & - & - & 48.3095 & 2.385 & 0.4053 \\
\hline Size biased Maxwell & - & - & - & - & 41.8373 & - & 0.9932 & 0.1647 \\
\hline Weighted Weibull (volume biased) & 0.0010038 & 2.1416 & 1.9372 & & & & 0.2039 & 0.0254 \\
\hline
\end{tabular}

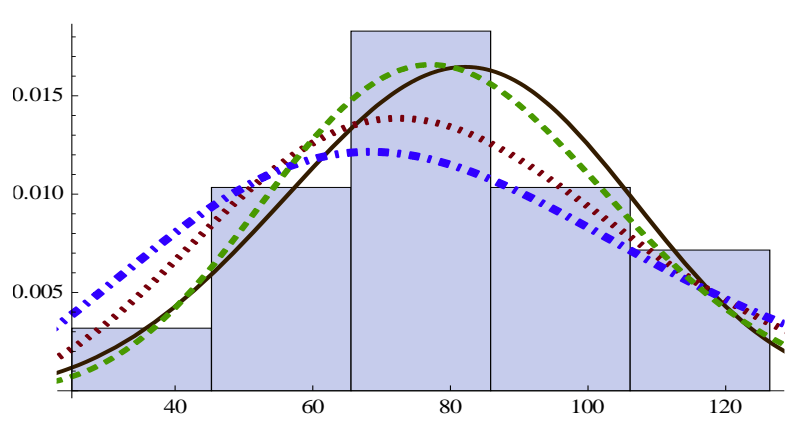

Fig. 6: Rayleigh (Spotted Line), Two parameter Weibull (Solid Line), Weighted Weibull (volume biased) Distribution (Dashed Line) and maxwell (Dotted Dashed) on the histogram

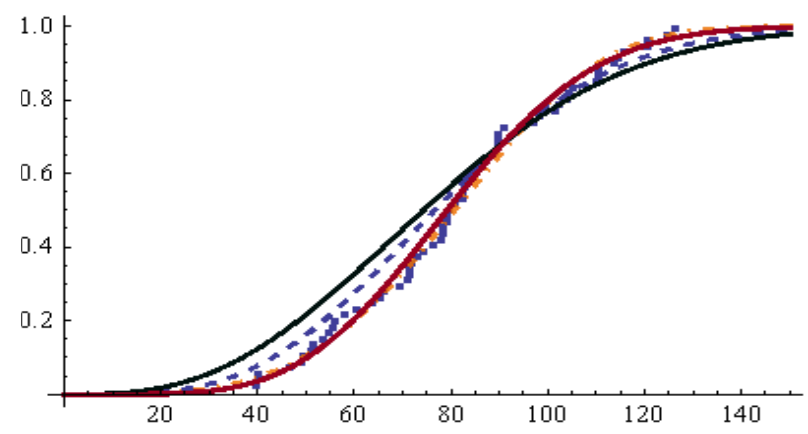

Fig. 7: Empirical cdf, weighted Weibull density estimates and cdf Estimates for the snowfall data

\section{Conclusion}

In this paper, we discussed the Volume Biased Weighted Weibull Distribution (VWWD). We discussed the statistical properties of this newly generated distribution in detail and obtained the mean, variance, moments, mode, coefficient of skewness, coefficient of kurtosis, reliability function, hazard function and the reverse hazard function. We have also provided results of entropies and characterization of VWWD. Also, the parameters of this distribution are estimated by the maximum likelihood estimation method. At the end, we have provided the comparison of VWWD with other wellknown distributions and it is well observed that VWWD fits better than those distributions.

\section{References}

Dara ST (2011). Recent Advances in moment distributions and their hazard rates. Ph.D. Dissertation, National College of Business Administration and Economics, Lahore, Pakistan.

Gradštejn IS and Ryžik IM (2007). Table of integrals, series, and products. Elsevier/Academic Press. Burlington, USA.

Nadarajah S and Kotz S (2004). The beta Gumbel distribution. Mathematical Problems in Engineering, 2004(4): 323-332.

Perveen Z, Ahmed Z, and Ahmad M (2016). On size-biased double weighted exponential distribution (SDWED). Open Journal of Statistics, 6(05), 917-930.

Provost S, Saboor A, and Ahmed M (2009). The gamma-Weibull distribution. Pakistan Journal of Statistics, 27(2): 111-131.

Silverman B (1986). Density estimation for statistics and data analysis. CRC press, London, UK. 